\title{
Cancer Chemoprevention
}

\author{
Amr Amin, ${ }^{1,2}$ Metka Filipič, ${ }^{3}$ Su S. Chen, ${ }^{4}$ and Regine Schneider-Stock ${ }^{5}$ \\ ${ }^{1}$ Biology Department, College of Science, UAE University, Al-Ain 17551, UAE \\ ${ }^{2}$ Zoology Department, Faculty of Science, Cairo University, Cairo 12613, Egypt \\ ${ }^{3}$ Department for Genetic Toxicology and Cancer Biology, National Institute of Biology, Večna Pot 111, Ljubljana, Slovenia \\ ${ }^{4}$ Department of Hematopathology, The University of Texas MD Anderson Cancer Center, Houston, TX 77030, USA \\ ${ }^{5}$ Experimental Tumor Pathology, Institute of Pathology, University of Erlangen, Erlangen, Germany
}

Correspondence should be addressed to Amr Amin, a.amin@uaeu.ac.ae

Received 20 September 2012; Accepted 20 September 2012

Copyright (C) 2012 Amr Amin et al. This is an open access article distributed under the Creative Commons Attribution License, which permits unrestricted use, distribution, and reproduction in any medium, provided the original work is properly cited.

Cancers are characterized by the dysregulation of cell signaling pathways at multiple steps. Most current anticancer therapies however involve the modulation of a single target. The lack of safety and high cost of monotargeted therapies have encouraged alternative approaches. Both natural compounds, extracted from plants or animals, and synthetic compounds, derived from natural prototype structures, are now being used as cancer therapeutics and as chemopreventive compounds.

Given the limited treatments available, preventive control approaches have been considered among the best strategies to protect against cancer. Chemoprevention takes advantage of the long latency of the disease that presents various opportunities for intervention. Chemopreventive intervention can cater to a defined population that is relatively at a higher risk for developing the disease with an objective to end, turn around, or slow down the progression of the disease. Exogenous phytochemicals are usually utilized to enhance endogenous mechanisms against various stages of cancer development.

Natural drugs have found direct medical application as drug entities, but they also serve as chemical models or templates for the design, synthesis, and semisynthesis of novel substances, such as paclitaxel (Taxol), vincristine (Oncovin), and camptothecin, in the treatment of human cancer. Although there are some new approaches to drug discovery, such as a combination of chemistry and computerbased molecular modeling design, none of them can replace the important role of natural products in drug discovery and development.

In this special issue, M. Cazzaniga and B. Bonanni review the current clinical research in both ER-positive and
ER-negative breast cancer chemoprevention, explaining the biologic effect of the various agents (such as: Tamoxifen, Raloxifene, Retinoids) on carcinogenesis and precancerous lesions, and finally present an excursus about new molecular targets under investigations in breast cancer settings. In one of the papers, C. Stolfi et al. highlight the available experimental data supporting the ability of mesalazine and its derivatives to interfere with intracellular signals involved in colorectal cancer cell growth.

Natural products encompass three main categories of compounds, phenylpropanoids, isoprenoids, and alkaloids, which are widely distributed in plant foods and medicinal herbs. This large array of molecules is crucial to human nutrition and health. Plant-derived foodstuffs and beverages also constitute the so-called functional foods and beverages, which include mainly fruits, vegetables, herbs, and spices. Curcumin is among the widely known spices that have been used in traditional medicine for many years and by different cultures. K. Sintara and his group in another paper here clearly show that Curcumin treatment for 20 weeks also decreased 8-hydroxy-2'-deoxyguanosine (8$\mathrm{OHdG}$ ) expression in benign tumor-bearing rats compared with N-methyl-N-nitrosourea and saturated sodium chloride. Curcumin can attenuate cancer via a reduction of phospho-I $\kappa \mathrm{B} \alpha$ and $8-\mathrm{OHdG}$ expressions, which may play a promising role in gastric carcinogenesis. In another paper, A. Amin and his colleagues have provided clear evidence that oral Ginkgo biloba (GB) administrations to cisplatintreated rats effectively alleviated cisplatin-induced toxicity in reproductive system. The present results provide further insights into the mechanisms of protection against cisplatininduced reproductive toxicity and confirm the essential 
antioxidant potential of a GB extract. Finally, A. Sapone et al. show that the idea that the physiological roles of specific catalysts may be easily manipulated by regular long-term administration of isolated nutrients and other chemicals derived from food plants is not viable. In contrast, it is the consumption of wholesome healthy diets that is most likely to reduce mutagenesis and cancer risk. They suggest that both research endeavors and dietary recommendations be redirected away from single molecules to dietary patterns as a main strategy for public health policy.

Amr Amin

Metka Filipič

Su S. Chen

Regine Schneider-Stock 


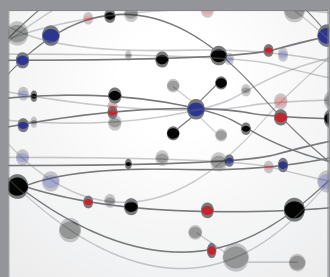

The Scientific World Journal
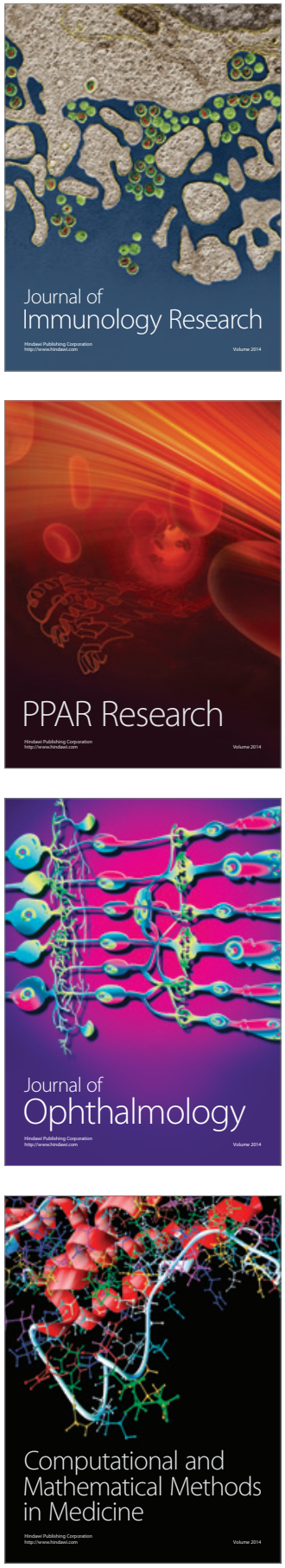

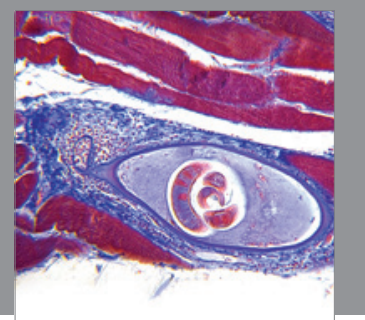

Gastroenterology

Research and Practice
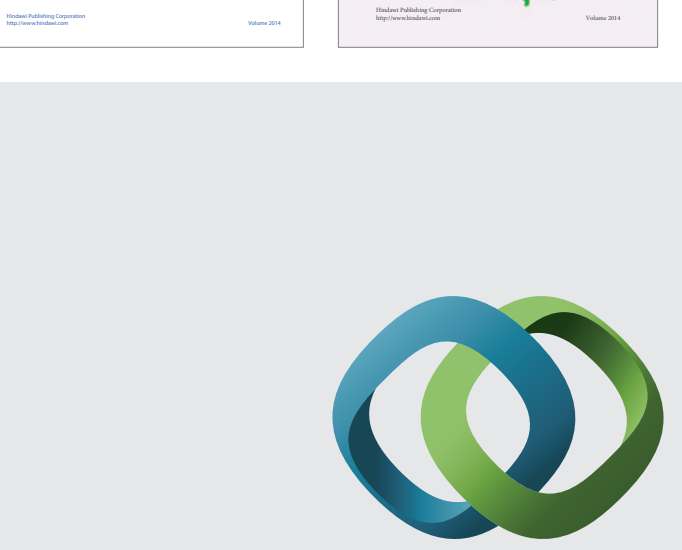

\section{Hindawi}

Submit your manuscripts at

http://www.hindawi.com
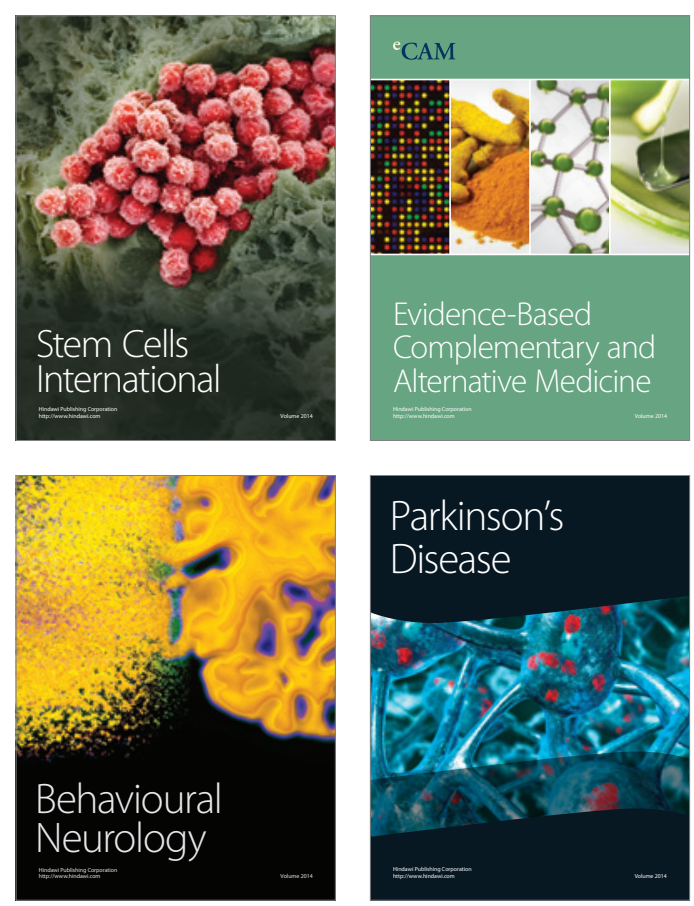

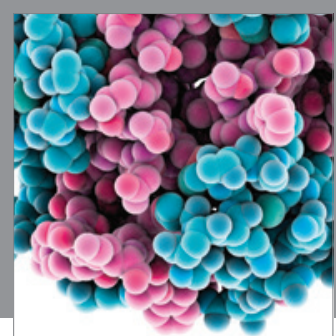

Journal of
Diabetes Research

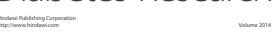

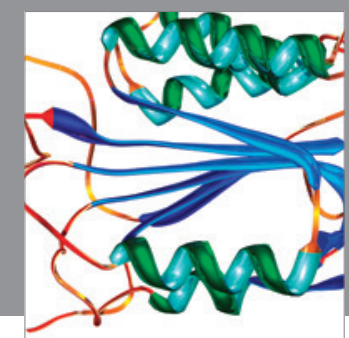

Disease Markers
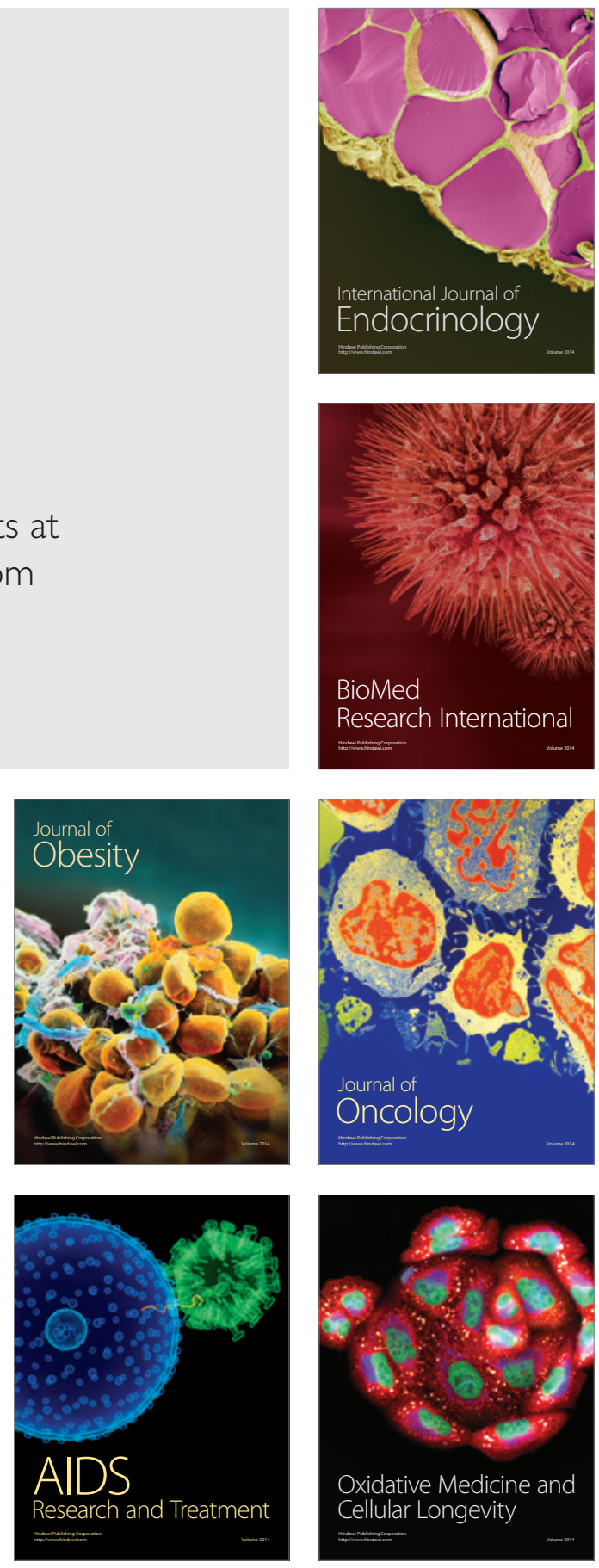